\title{
A Comparative Study of All Optical Signal Regeneration Techniques
}

\author{
Md. Ziaul Amin and Khurram Karim Qureshi*
}

Electrical Engineering Department, King Fahd University of Petroleum and Minerals, Dhahran 31261, Saudi Arabia

\begin{abstract}
An efficient optical signal regenerator can mitigate the effect of signal degradation and increase the performance of optical communication system. All optical signal regenerators have been studied and developed for maintaining good quality signal in long haul optical communication. Fiber nonlinearity has some inherent properties for signal regeneration and it has an enormous potential for ultra-high speed optical communication. In this paper, all optical signal regeneration techniques using self-phase modulation (SPM) are examined. Major drawbacks of the existing regenerators have also been identified.
\end{abstract}

Keywords: All optical signal regenerator, Fiber nonlinearity, Self-phase modulation (SPM).

\section{INTRODUCTION}

Conventionally in optical communication system, signal regeneration is performed by optical-electrical-optical (OEO) conversion. This conversion process puts limit on transmission data rate of the information signal. There is another type of regeneration which does not depend on OEO conversion. In this method, signal regeneration is undertaken directly in the optical domain and is known as all optical signal regeneration. This mechanism can process high speed signal with less power consumption. In last two decades, a number of all optical signal regeneration schemes have been reported [14].

Signal propagation through the optical fiber is distorted by different sources. These sources can be divided into two types such as deterministic and stochastic. Deterministic signal distortion can be mitigated by electrical or optical means [5]. On the other hand, signal degradation caused by stochastic process cannot be mitigated deterministically. After the mitigation of deterministic signal distortion, the maximum transmission link will depend on the accumulation of the stochastic noise. Optical regenerator can mitigate the effect of this random noise and increase the transmission distance.

For designing a high capacity link, optical nonlinearity can be a major limitation. On the other hand, this optical nonlinearity can be used in different useful applications like wavelength conversion, pulse regeneration, optical signal monitoring and switching [6]. The next generation all optical networks depend strongly on the use of nonlinear properties. All optical signal regenerators using nonlinear property have been developed rapidly and need further investigations.

*Address correspondence to this author at the Electrical Engineering Department, King Fahd University of Petroleum and Minerals, Dhahran 31261, Saudi Arabia; Tel: 009668603846; E-mail: kqureshi@kfupm.edu.sa
Different nonlinear effects such as self-phase modulation (SPM), cross phase modulation (XPM), four waves mixing (FWM), and stimulated Raman scattering can be used for all optical signal regeneration [7, 8]. However, due to the advantages of SPM, it is mostly used in all optical signal regeneration. In this type of regeneration scheme neither pump sources nor probe source are required. Therefore, all optical signal regeneration using SPM is much simpler than XPM or FWM based regenerators.

In this paper, fiber based all optical signal regeneration methods using SPM is presented. In section II, Kerr and saturating nonlinearity are introduced. In section III, self- phase modulation and soliton formation are discussed. Different pulse regeneration schemes are described in section IV. Finally, section V concludes the paper.

\section{OPTICAL NONLINEARITY}

\subsection{Kerr Nonlinearity}

Intensity dependent refractive index of the fiber materials and inelastic scattering phenomenon are responsible for fiber non-linearity. In the intense electric field, materials cannot provide linear response. Polarization of dielectric medium due to applied electric field, $E$ can be expressed by [9]:

$P=\epsilon_{0} \chi^{(1)} E+\epsilon_{0} \chi^{(2)} E^{2}+\epsilon_{0} \chi^{(3)} E^{3}+\cdots$

Where $\epsilon_{0}$ is the permittivity of the vacuum and $\chi^{(k)}$ $\left(k=1,2,3^{\cdots}\right)$ is the $k_{\text {th }}$ order susceptibility. First order susceptibility $\left(\chi^{(1)}\right)$ and second order susceptibility $\left(\chi^{(2)}\right)$ are responsible for linear and second harmonic generation respectively. Third order susceptibility $\left(\chi^{(3)}\right)$ introduces lowest order nonlinear effect in the fiber [10]. For the sinusoidal variation of the applied electric field, $E=E_{0} \cos (\omega t-k z)$ polarization vector can be written as: 
$P=\epsilon_{0} \chi^{(1)} E_{0} \cos (\omega t-k z)+\epsilon_{0} \chi^{(2)} E_{0}{ }^{2} \cos ^{2}(\omega t-k z)+$ $\epsilon_{0} \chi^{(3)} E_{0}{ }^{3} \cos ^{3}(\omega t-k z)+\cdots$

By applying some trigonometric identities (2) can be expressed as:

$P=$

$\frac{1}{2} \epsilon_{0} \chi^{(2)} E_{0}{ }^{2}+\epsilon_{0} \chi^{(1)} E_{0} \cos (\omega t-k z)+$

$\frac{3}{4} \epsilon_{0} \chi^{(3)} E_{0}{ }^{2} E_{0} \cos (\omega t-k z)+\frac{1}{2} \epsilon_{0} \chi^{(2)} E_{0}{ }^{2} \cos 2(\omega t-$

$k z)+\frac{1}{4} \epsilon_{0} \chi^{(3)} E_{0}{ }^{3} \cos 3(\omega t-k z)$

For the practical optical fiber, $\chi^{(2)}=0$

Therefore, (3) can be written as:

$P=\epsilon_{0} \chi^{(1)} E_{0} \cos (\omega t-k z)+\frac{3}{4} \epsilon_{0} \chi^{(3)} E_{0}{ }^{2} E_{0} \cos (\omega t-$

$k z)+\frac{1}{4} \epsilon_{0} \chi^{(3)} E_{0}{ }^{3} \cos 3(\omega t-k z)+\cdots$

Due to variations in refractive index of the fiber there is lack of phase between frequencies $\omega$ and $3 \omega$. Therefore, the third term of (4) can be neglected due to this phase mismatch. Higher order terms of (4) can also be neglected due to less contribution. Equation (4) can be expressed in terms of intensity, $I=\frac{1}{2} c \epsilon_{0} n_{l} E_{0}^{2}$ as:

$P=\epsilon_{0} \chi^{(1)} E_{0} \cos (\omega t-k z)+\frac{3}{2} \frac{\chi^{(3)}}{c n_{l}} I E_{0} \cos (\omega t-k z)$

Where, $c$ and $n_{l}$ are the velocity of light and linear refractive index of the medium respectively.

Effective susceptibility $\chi_{e f f}$ and refractive index $n_{e f f}$ can be defined as:

$\chi_{\text {eff }}=\frac{P}{\epsilon_{0} E}=\chi^{(1)}+\frac{3}{2} \frac{\chi^{(3)}}{c \epsilon_{0} n_{l}} I$

$n_{\text {eff }}=\left(1+\chi_{e f f}\right)^{\frac{1}{2}}=\left(1+\chi^{(1)}+\frac{3}{2} \frac{\chi^{(3)}}{c \epsilon_{0} n_{l}} I\right)^{\frac{1}{2}}$

By using Taylor's series expansion $n_{\text {eff }}$ can be approximated as:

$n_{\text {eff }}=n_{l}+\frac{3}{4} \frac{\chi^{(3)}}{c \epsilon_{0} n_{l}} I=n_{l}+n_{n l} I$

Where $n_{l}=\left[1+\chi^{(1)}\right]^{\frac{1}{2}}$ and $n_{n l}=\frac{3}{4} \frac{\chi^{(3)}}{c \epsilon_{0} n_{l}}$

Here $n_{n l}$ is the Kerr coefficient.

In the Kerr nonlinear medium, the index change $\Delta n$ is linearly proportional to the optical intensity $I$, i.e., $\Delta n(I)=n_{n l} I$.

Therefore, a dielectric medium shows a nonlinear behavior, when high intensity light pulse is transmitted through that medium.

\subsection{Saturable Nonlinearity}

In the Kerr nonlinear medium, the refractive index change is proportional to the optical intensity. However, there exist materials in which intensity dependent refractive index change has a maximum limit (i.e., the intensity dependent dielectric constant saturate to a certain value). The prominent effect of saturation is found in materials with high nonlinear susceptibility $\chi^{(3)}$, such as fluorescein-doped acid glass, which has a $\chi^{(3)}$ value as large as $1 \mathrm{esu}$, which is $10^{15}$ times larger than that of silica and $10^{13}$ times larger than that of $\mathrm{CS}_{2}$. The advantages of a saturable nonlinear medium include beam tapering and uptapering by using gain and absorption of the medium. Moreover, this medium has been proven to be a key to many of the new families of solitons discovered in the 1990s [11-14].

\section{SELF-PHASE MODULATION}

Due to the nonlinear property of the fiber, different portions of the optical pulses encounter different refractive indices depending on the intensity of the pulse. If an optical pulse is transmitted through the fiber, the higher intensity portion of the pulse encounters a higher refractive index as compared to the lower intensity portion [9]. The front end of the pulse faces positive refractive index gradient $\frac{d n}{d t}$ and back end of the pulse faces negative refractive index gradient $-\frac{d n}{d t}$, as shown in Fig. (1). This changing refractive index results temporally varying phase changes. The phase change of different portions of the pulses is different depending on the intensity of the pulse. This phase modulation of the optical pulse occurs due to varying intensity of the pulse itself and nonlinear behavior of the fiber. This phase modulation phenomenon is known as self-phase modulation.

The phase $\varphi$ introduced by a field, $(E)$ can be expressed by the following relation [15]:

$\varphi=\frac{2 \pi}{\lambda} n L$

Where, $n, L$ and $\lambda$ are refractive index, fiber length and propagation wavelength respectively.

If the fiber contains high transmitted power, the phase information can be expressed by the following relation:

$\varphi=\frac{2 \pi}{\lambda} n_{e f f} L_{e f f}=\frac{2 \pi}{\lambda}\left(n_{l}+n_{N l} I\right) L_{e f f}$

Where, first and second term of the above equation represent the linear and nonlinear portion of the phase constant. Time dependent pulse intensity is responsible for time dependent phase [16]. Consequently, this phase variation with respect to time modify the frequency spectrum, which can be presented as:

$\omega=\frac{d \varphi}{d t}$

In a dispersive medium, a change in the spectrum of temporally varying pulse will change the nature of the variation. This variation nature can be shown by considering a Gaussian pulse, which modulates an optical carrier frequency, $\omega_{0}$ and creats a new instantanious frequency:

$\omega^{\prime}=\omega_{0}+\frac{d \varphi}{d t}$ 


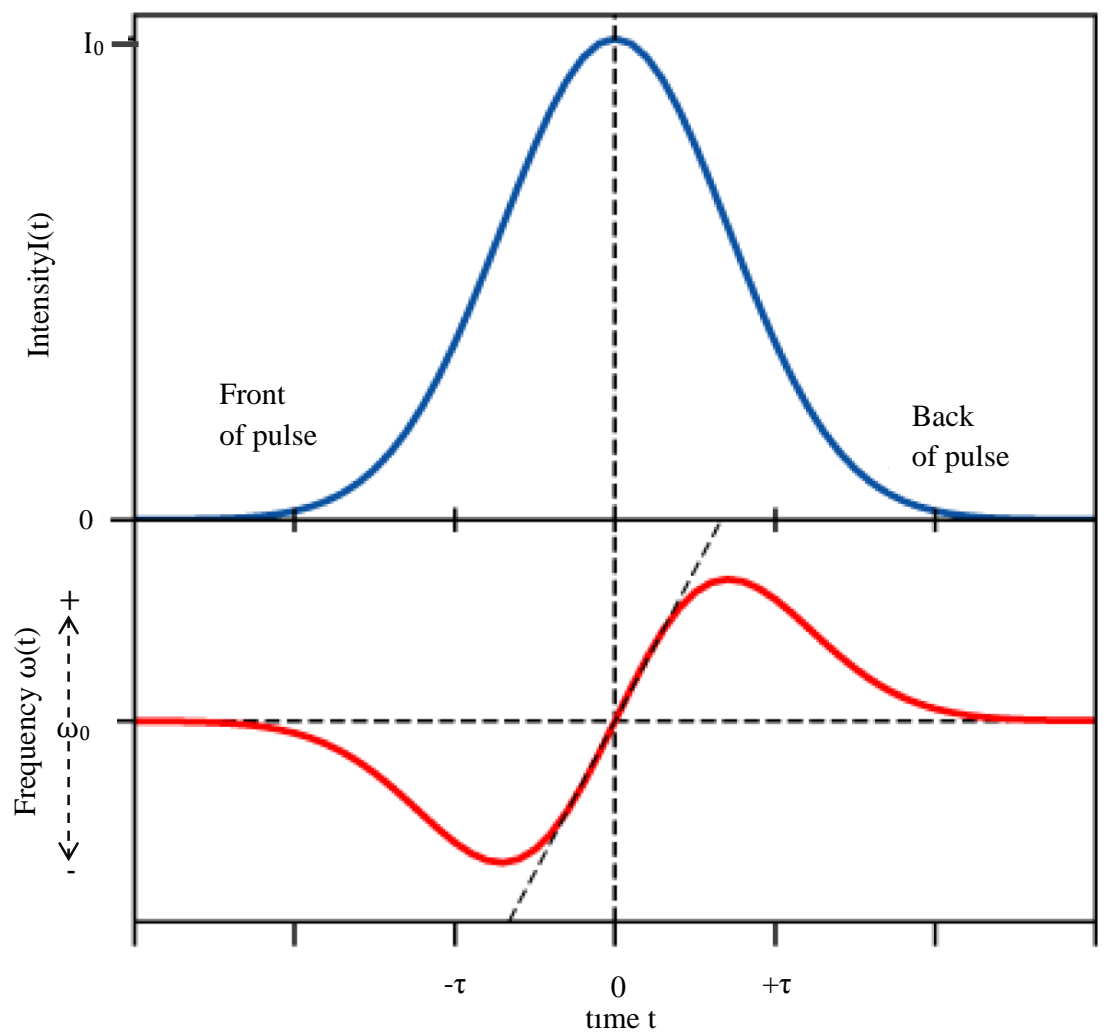

Fig. (1). SPM introduced pulse broadening [15].

Phase change for SPM effect will be negative because of minus sign in the phase $(\omega t-k z)$ expression. Therefore, equation (12) can be written in the following form:

$\omega^{\prime}=\omega_{0}-\frac{2 \pi}{\lambda} n_{N l} L_{e f f} \frac{d I}{d t}$

For the front end of the pulse, $\frac{d I}{d t}>0$ hence

$\omega^{\prime}=\omega_{0}-\omega(t)$

On the other hand, for the back end of the pulse $\frac{d I}{d t}<0$

$\omega^{\prime}=\omega_{0}+\omega(t)$

Equation (14) and (15) indicate that SPM results in frequency chirping. This leads to the spectral broadening without any change in temporal distribution. Lower and higher frequency chirping are found in front and back end of the pulse respectively, as shown in Fig. (1).

On the other hand, the fiber used in the linear dispersion region, wavelength above zero dispersion, creates opposite chirping effect of SPM. This opposite chirping effect can be balanced by proper choice of pulse shape (a hyperbolic secant-shape) and pulse power. Under this situation, pulse will propagate along the fiber without broadening in the time and frequency domains and known as temporal soliton [17, 18]. There is another form of soliton known as spatial soliton that propagates in a nonlinear medium without diffraction. This happens due to the exact balance between diffraction and nonlinearly induced self-focusing effects [11].

\section{SIGNAL REGENERATION}

\subsection{Regenerator Based on Power-Dependent Spectrum Broadening and Offset Filtering}

Spectralwidth of an optical pulse that is to be regenerated is expressed as $\Delta \omega_{0} \sim \frac{1}{\tau}$ ( $\tau$-pulse width) [19]. This spectrum of the pulse will be broaden due to SPM effect. The broadened pulse width is given by:

$\Delta \omega_{S P M}=\Delta \omega_{0}\left(\frac{2 \pi}{\lambda}\right) n_{N l} I$

After bandpass filtering of the broadened pulse, input signal frequency $\left(\omega_{0}\right)$ will be shifted according to the following relation:

$\omega_{f}=\omega_{0}+\Delta \omega_{\text {Shift }}$

Where, $\omega_{f}$ is the center frequency of the band pass filter.

For the small spectral broadening of the pulse

$\frac{\omega_{S P M}}{2}<\Delta \omega_{\text {Shift }}$

The pulse will be rejected by the filter. This situation occurs when the intensity of the pulse is very small ( noise in "zeros"). On the other hand spectral brodening for high intensity pulse follows the relation (19). Here the spectralwidth of the pulse will be determined by the filter spectralwidth $\Delta \omega_{f}$. 


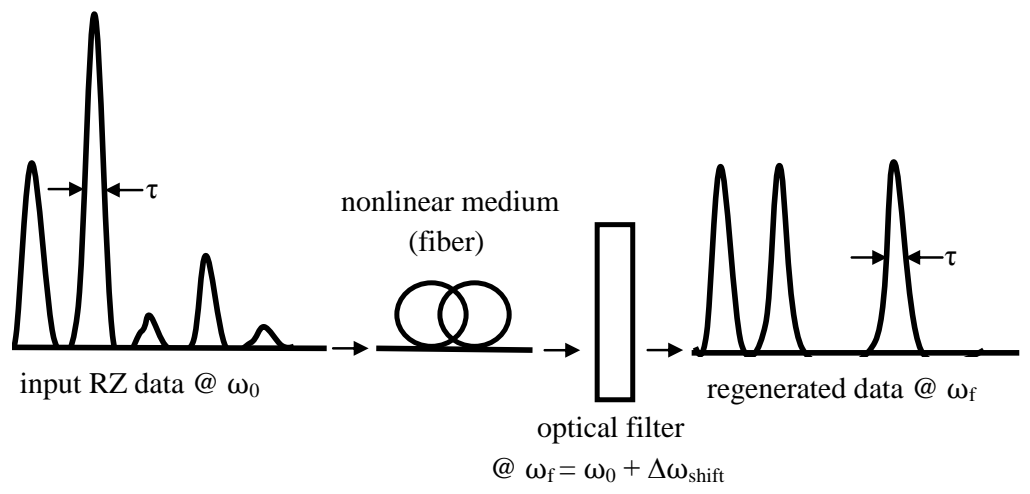

Fig. (2). Regenerator based on spectrum broadening and offset filtering [19].

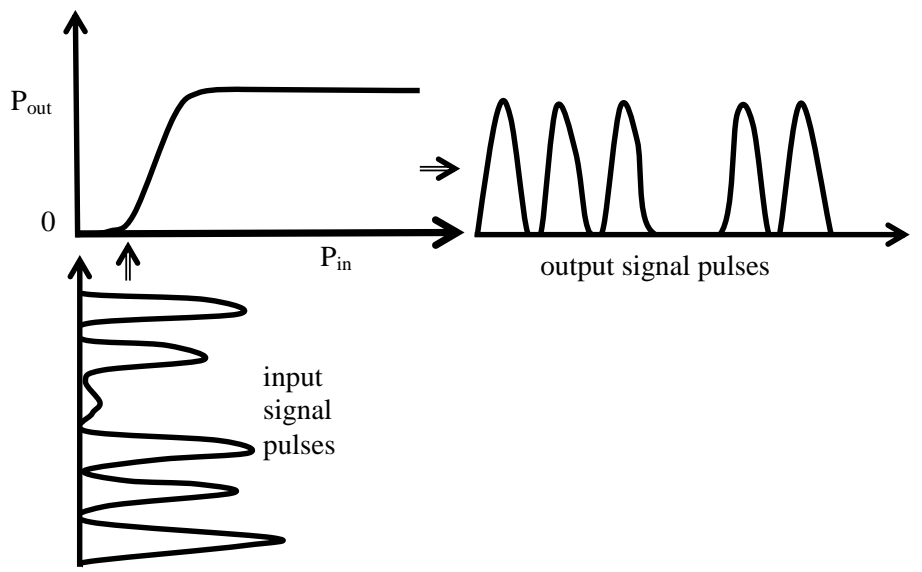

Fig. (3). Power transfer curve for power dependent spectrum broadening and spectrum filtering regenerator [20].

$\frac{\omega_{S P M}}{2} \geq \Delta \omega_{\text {Shift }}$

Thererfore, output pulse width can be changed by changing the filter spectral bandwidth $\Delta \omega_{f}$. For the case; $\Delta \omega_{f} \sim \Delta \omega_{0}$, the output pulse width will be same as the input pulse width. After spectral filtering, the output pulse intensity is proportional to the spectral density of the SPM broadened spectrum, $I_{\omega} \equiv \frac{d I}{d \omega}$.

The following relation can be estimated using (16):

$I_{\omega} \sim \frac{I_{p}}{\Delta \omega_{S P M}}=\frac{\lambda}{\Delta \omega_{0} 2 \pi n_{N l} L}$

The above expression shows that $I_{\omega}$ and consequently the output pulse intensity are not dependent on the input pulse intensity $I_{p}$.

If the relation (19) is met for high $I_{p}$, pulse transfer function of the regenerator of Fig. (2) can be expressed as:

$I_{\text {out }}=0$ if $I_{p}<I_{C R}$

$I_{\text {out }}=$ Constant if $I_{p}>I_{C R}$

Where $I_{C R}$ is the critical pulse intensity, which can be determined by:

$\frac{\Delta \omega_{S P M}\left(I_{C R}\right)}{2}=\Delta \omega_{\text {Shift }}$
$I_{C R}=\frac{2 \Delta \omega_{\text {Shift }}}{\Delta \omega_{0}(2 \pi / \lambda) n_{2} L}$

Transfer function represented by (21a) and (21b) is shown in Fig. (3). This step like nonlinear transfer fuction is realized by the use of SPM effect in the fiber and subsequent filtering [20]. This nonlinear power transfer curve ensures the zero level noise suppression and amplitude level signal fluctuation.

The noise suppression can be further improved by using cascaded stages of this regenerator [21]. Moreover, multiple stages can provide wavelength shift free signal regeneration [22]. Two cascaded stages as shown in Fig. (4) are used for getting back the signal frequency or wavelength at original value [20]. For satisfying this objective, the center wavelength of the first $\left(\lambda_{1}\right)$ and second $\left(\lambda_{2}\right)$ OBPFs should be selected such that the following conditions are met:

$\lambda_{1} \neq \lambda_{2}, \lambda_{2} \neq \lambda_{3}$ and $\lambda_{1}=\lambda_{3} \neq \lambda_{2}$

In a cascaded regenerator of Fig. (4), it is often difficult to prepare two spools of nonlinear fibers, which will satisfy the signal regenerator requirement. Therefore, bidirectional configuration, as shown in Fig. (5), is proposed in [23], where signal will be transmitted twice along the same nonlinear fiber in opposite direction. This bidirectional two stage configuration can reduce the number of nolinear fibers from two to one. This also shows strong improvement in 


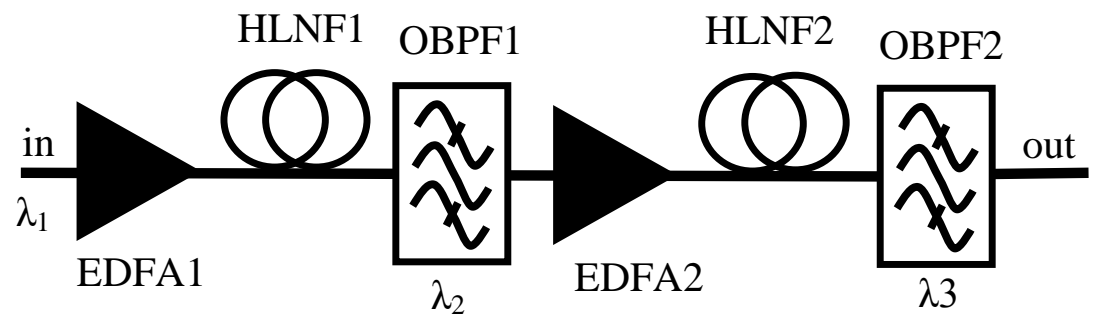

Fig. (4). 2-R Cascaded SPM based regenerator [24].

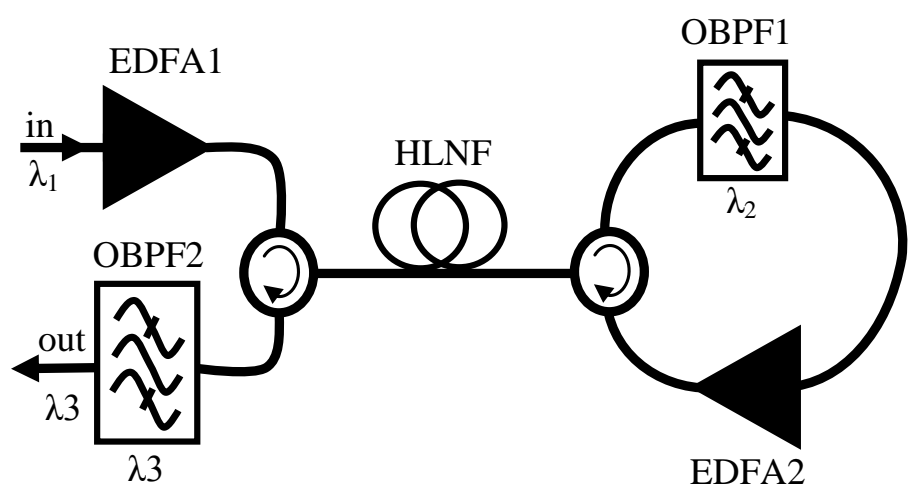

Fig. (5). 2-R bidirectional SPM based regenerator [24].

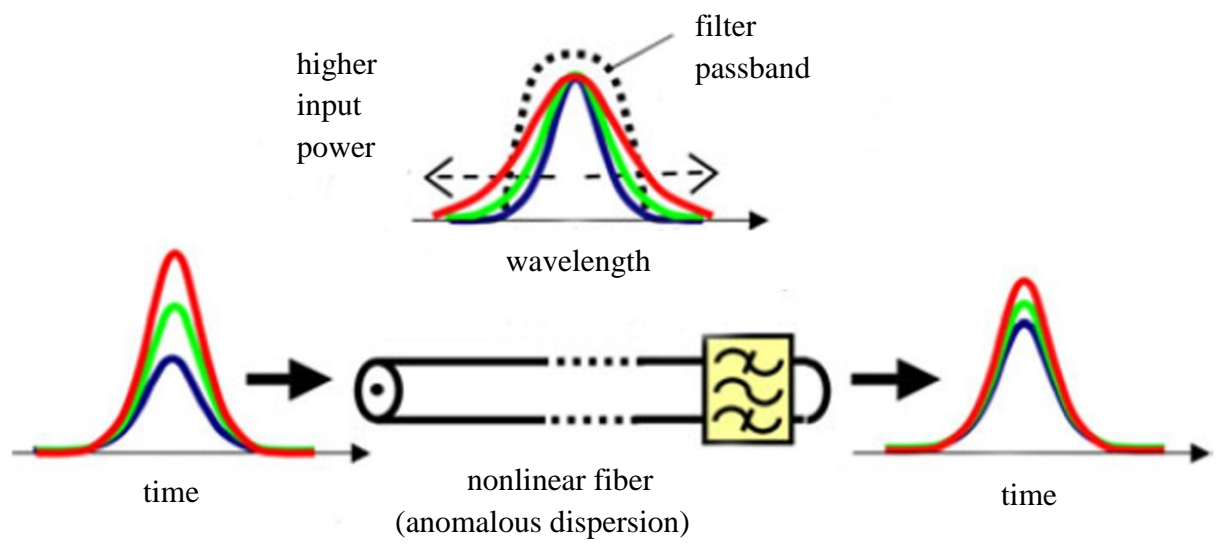

Fig. (6). Pulse regeneration using soliton filtering [5].

extinction ratio. However, one major concern with this configuration is the influence of Rayleigh Backscattering (RB) in the HNLF. This RB will act as noise to the signal travelling in the oppsite direction.

\subsection{Regenerator Based on Solitonic filtering}

Another SPM based regenerator can be constructed similar to that described in section IV-A. Here, the nonlinear fiber with anomalous dispersion is selected such that it supports soliton propagation. The input pulse propagating through the Highly Nonlinear Fiber (HLNF) encounters temporal compression or decompression depending on whether its amplitude is larger or smaller than that of the fundamental soliton [25]. Spectral width of the pulse will be broadened or narrowed down for adiabetic pulse compression or decompression.

In this type of regenerator, a bandpass filter with fixed bandwidth is used after the nonlinear fiber as shown in Fig. (6), for applying larger (smaller) energy loss to the pulse having larger (smaller) initial apmlitude at the input of the fiber. Therefore, automatically stabillized output is obtained using this method. This stabillized output can be maintained with moderate spectrum broadening as compared with the regenerator described in IV-A. Hence, input pulse can be smaller than the previous method. A problem associated with this method is that noise components remain in the same 


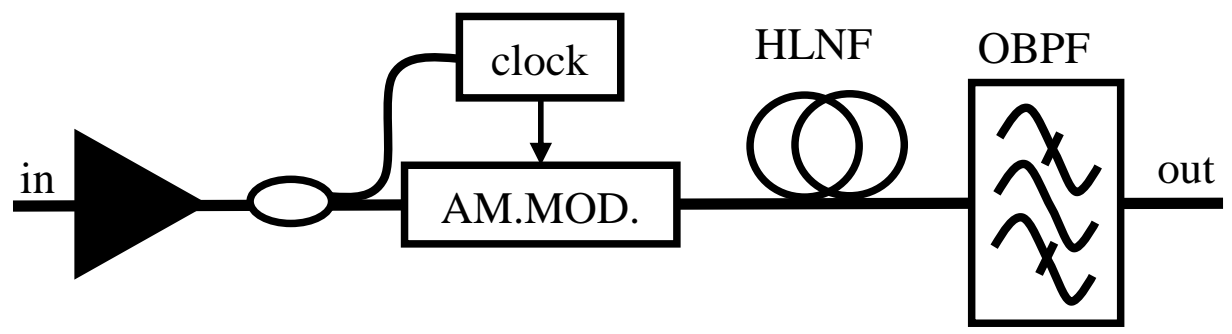

Fig. (7). 3-R regenerator using amplifier, synchronous modulator, HNLF and OBPF [5].

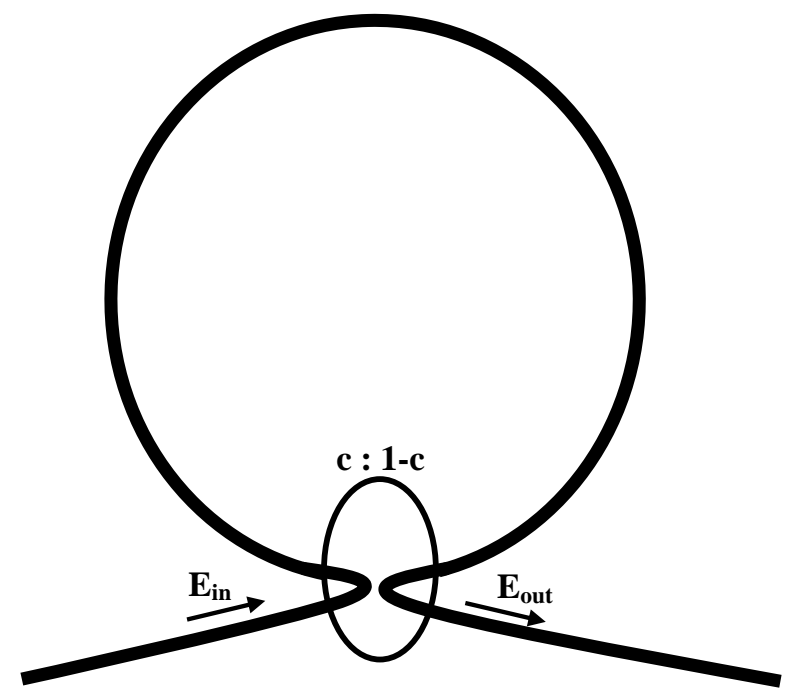

Fig. (8). Nonlinear optical loop mirror (NOLM) [5].

wavelength range as the signal is amplified by an excess gain around the center frequency of the filter. If the cascaded stages are used noise will be built up. By using satuarable absorbing element, this noise growth can be suppressed [26]. This problem can also be minimized by adding a synchronous amplitude modulator as shown in Fig. (7).

\subsection{Regenerator Using Nonlinear Interferometer}

In nonlinear interferometer, transmission coefficient can be changed by using the input signal power. This property is used in the signal regeneration mechanism.Though the interferometer can be designed in different forms but nonlinear optical loop mirror (NOLM) is extensively used for all optical signal regeneration. Doran and Wood proposed NOLM in [27], which is actually an optical directional coupler and the two output ports of this directional coupler are connected together by a fiber loop, as shown in Fig. (8). In this device, a single input is split into two counterpropagating fields, which return in coincidence to recombine at the coupler. In the linear medium, the split signals follow the same optical path but in the opposite direction. In the nonlinear medium the phase velocity is intensity dependent; therefore, propagation effects will no longer be identical for the two paths if $c \neq 0.5$. The phase shift acquired by a field $E_{\text {in }}$ propagating through the fiber loop under the influence of SPM effects is given by the following relation: $\varphi=\frac{2 \pi n_{N l}\left|E_{i n}\right|^{2} L}{\lambda}$

The output value at port 2 has been deduced in [27] by considering a single input at port 1 . The output value at port 2 is given by the following equation:

$\left|E_{\text {out }}\right|^{2}=$
$\left|E_{\text {in }}\right|^{2}\left\{1-2 c(1-c)\left[1+\cos \left[(1-2 c) \frac{2 \pi n_{N l}\left|E_{\text {in }}\right|^{2} L}{\lambda}\right]\right]\right\}$

Where $E_{\text {in }}, E_{\text {out }}$, and $L$ are the input power, output power, and length of the loop fiber respectively. From (25), the transmitivity of the fiber can be written by the following expression:

$$
\begin{aligned}
& T=\frac{\left|E_{\text {out }}\right|^{2}}{\left|E_{\text {in }}\right|^{2}}=e^{-\alpha L}\{1-2 c(1-c)[1+\cos [(1- \\
& \left.\left.\left.2 c) \frac{2 \pi n_{N l}\left|E_{\text {in }}\right|^{2} L}{\lambda}\right]\right]\right\}
\end{aligned}
$$

Here, lossy fiber with loss coefficient $\alpha$ is considered as the loop.

For getting the power dependent output, the symmetry of the loop needs to be broken. This can be done by setting $c \neq 0.5$ in Fig. (8). From (25), output power can be made constant by setting the input power at one of the values. It is also clear from (26) that transmittivity ( $T_{\min }=e^{-\alpha L}[1-$ $4 c(1-c)]$ ) becomes minimum with the vanishing input. 


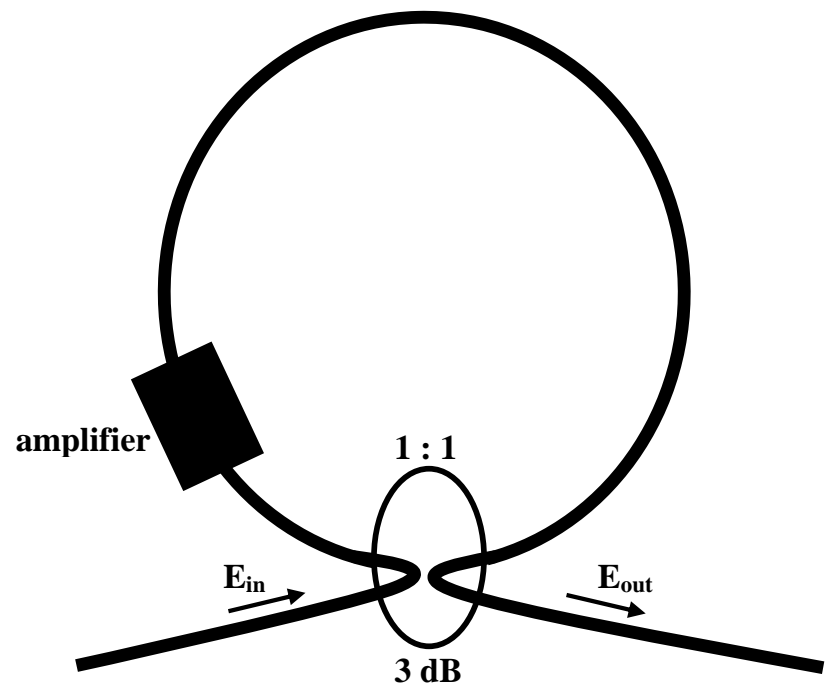

Fig. (9). Nonlinear amplifying loop mirror (NALM) [5].

Therefore, NOLM can suppress the zero level noise [28, 29]. By controlling coupling ratio c, better zero-level noise supression and higher contrast in output power can be obtained. Coupling ratio (c) closer to 0.5 is usually selected for better results. The transmittivity relation of NOLM uses input signal at every instance of time.

In NOLM, different portions of the pulses will be transmitted differently for the pulse input. This leads to the partial transmission of the pulse and pulse breakup. By using solitonic effects in NOLM, the optical pulse can be transmitted as a whole and better switching can be obtained [27]. Another variant of the NOLM is the nonlinear amplifying loop mirror (NALM). In NALM, a bidirectional amplifier is used at one end of fiber loop to break the symmetry of the loop, as shown in the Fig. (9) [30]. The directional coupler may couple even power. Power transmission ratio of the NALM can be given by the following relation [5]:

$T=\frac{\left|E_{\text {out }}\right|^{2}}{\left|E_{\text {in }}\right|^{2}}=G e^{-\alpha L} \sin ^{2}\left[\frac{\gamma\left|E_{\text {in }}\right|^{2} L_{e f f}(G-1)}{4}\right]$

Where, $G$ is the gain of the amplifier.

In NALM, loss element can be used instead of bidirectional amplifier for the same function. But output power is attenuated [28]. Transmission ratio equation of NALM shows that output power $\left|E_{\text {out }}\right|^{2}$ is proportional to the third power of the input power $\left|E_{i n}\right|^{2}$, when input power is small. Therefore, NALM has better ability of zero-level noise suppression than NOLM.

\section{CONCLUSION}

All optical signal regeneration can overcome the distance limitations caused by the noise accumulation in ultra-high speed optical communication. These regeneration techniques use fiber nonlinearity which requires isolated optical pulses. The regeneration performance is severely deteriorated if the pulses are temporarily overlapping with each other. This indicates that optical fiber must be precisely dispersion compensated at the regenerator location. Moreover, in WDM system the channel must be de-multiplexed before regeneration operation. These undesired features reduce flexibility and increase the system cost. Multichannel all optical signal regeneration is still a challenge since satisfactory performance has not been reported yet.

\section{CONFLICT OF INTEREST}

The authors confirm that the content of this review has no conflict of interest.

\section{ACKNOWLEDGEMENTS}

Declare None.

\section{REFERENCES}

[1] Jinno M, Abe M. All-optical regenerator based on nonlinear fiber sagnac interferometer. Electron Lett 1992; 28(14): 1350-2.

[2] Lucek JK, Smith K. All-optical signal regenerator. Opt Lett 1993; 18(15): 1226-8.

[3] Leclerc O, Lavigne B, Chiaroni D, Desurvire E. All-optical regeneration: principles and WDM implementation. In: Kaminow IP, Tingye L, Eds. Optical fiber telecommunications iv-a: components. CA: Academic Press 2002; pp. 732-83.

[4] Simon JC, Gay M, Bramerie L, et al. Long distance transmission using optical regeneration. In: Optical Fiber Communications Conference, 24-28 Feb. San Diego, CA, 2008; pp. 1-3.

[5] Matsumoto M. Fiber-based all-optical signal regeneration. IEEE J Sel Top Quantum Electron 2012; 18(2): 738-52.

[6] Toulouse J. Optical nonlinearities in fibers: review, recent examples, and systems applications. J Light Technol 2005; 23(11): 3625-41.

[7] Agrawal GP. Applications of nonlinear fiber optics. $2^{\text {nd }}$ ed. San Francisco, CA: Academic Press 2008.

[8] Parmigiani F, Asimakis S, Sugimoto N, Koizumi F, Petropoulos P, Richardson DJ. 2R regenerator based on a 2-m-long highly nonlinear bismuth oxide fiber. Opt Express 2006; 14(12): 5038-44. 
[9] Singh SP, Singh N. Nonlinear effects in optical fibers: origin, management and applications. Prog Electromagn Res 2007; 73:249-75

[10] Agrawal GP. Nonlinear fiber optics. $3^{\text {rd }}$ ed. San Diego, CA: Academic Press 2001

[11] Stegeman GIA, Christodoulides DN, Segev M. Optical spatial solitons: historical perspectives. IEEE J Sel Top Quantum Electron 2000; 6(6): 1419-27.

[12] Chen Y. Self-trapped light in saturable nonlinear media. Opt Lett 1991; 16(1): 4-6.

[13] Konar S, Sengupta A. Propagation of an elliptical Gaussian laser beam in a medium with saturable nonlinearity. J Opical Soc Am B 1994; 2(1): 2-5.

[14] Sodha MS, Medhekar S, Konar S, Saxena A, Rajkamal R. Absorption/amplification-induced self-tapering and uptapering of a laser beam in a saturable nonlinear medium: large nonlinearity. Opt Lett 1994; 19(15): 1110.

[15] Gangwar R, Singh SP, Singh N. Soliton based optical communication. Prog Electromagn Res 2007; 74: 157-66.

[16] Chraplyvy AR, Marcuse D, Henry PS. Carrier induced phase noise in angle-modulated optical fiber systems. J Light Technol 1984; 2(1): 6-10.

[17] Haus HA. Optical fiber solitons: their properties and uses. Proc IEEE 1993; 81(7): 970-83.

[18] Biswas A, Konar S. Soliton-soliton interaction with kerr law nonlinearity. J Electromagn Waves Appl 2005; 19(11): 1443-53.

[19] Mamyshev PV. All-optical data regeneration based on self-phase modulation effect. In: European Conference on Optical Communications 1998; pp. 475-6.

[20] Matsumoto M. All-optical signal regeneration using fiber nonlinearity. Eur Phys J Spec Top 2009; 173: 297-312.
[21] Matsumoto M. Performance comparison of all-optical signal regenerators utilizing self-phase modulation in fiber. In: Optical Fiber Communications Conference 2003; pp. 108-9.

[22] Raybon G, Su Y, Leuthold J, et al. $40 \mathrm{Gbit} / \mathrm{s}$ pseudo-linear transmission over one million kilometers. In: Optical Fiber Communication Conference 2002.

[23] Matsumoto M. Efficient all-optical 2R regeneration using selfphase modulation in bidirectional fiber configuration. Opt Express 2006; 14(23): 11018-23.

[24] Matsumoto M, Shimada Y, Sakaguchi H. Two-stage SPM based all-optical $2 \mathrm{R}$ regeneration by bidirectional use of a highly nonlinear fiber. IEEE J Quantum Electron 2009; 45(1): 51-7.

[25] Ohara T, Takara H, Hirano A, Mori K, Kawanishi S. 40-Gb/s $\times 4$ channel all-optical multichannel limiter utilizing spectrally filtered optical solitons. IEEE Photonics Technol Lett 2003; 15(5): 763-5.

[26] Matsumoto M, Leclerc O. Analysis of a 2R optical regenerator utilizing self-phase modulation in a highly nonlinear fiber. In: Lasers and Electro-Optics Japan: Osaka University 2002. pp. 6134.

[27] Doran NJ, Wood D. Nonlinear-optical loop mirror. Opt Lett 1988; 13(1): 56-8.

[28] Smith K, Doran NJ, Wigley PG. Pulse shaping, compression, and pedestal suppression employing a nonlinear-optical loop mirror. Opt Lett 1990; 15(22): 1294-6.

[29] Yamada E, Nakazawa M. Reduction of amplified spontaneous emission from a transmitted soliton signal using a nonlinear amplifying loop mirror and a nonlinear optical loop mirror. IEEE J Quantum Electron 1994; 30(8): 1842-50.

[30] Fermann ME, Haberl F, Hofer M, Hochreiter $H$. Nonlinear amplifying loop mirror. Opt Lett 1990; 15(13): 752-4.

(C) Amin and Qureshi; Licensee Bentham Open.

This is an open access article licensed under the terms of the Creative Commons Attribution Non-Commercial License (http://creativecommons.org/licenses/by$\mathrm{nc} / 3.0 /$ ) which permits unrestricted, non-commercial use, distribution and reproduction in any medium, provided the work is properly cited. 